\title{
Échoendoscopie et coloscopie en France : formation et qualité
}

\author{
Echoendoscopy and Colonoscopy in France: Training and Quality
}

\author{
E. Bories \\ (C) Lavoisier SAS 2016
}

Le congrès annuel du club francophone d'échoendoscopie, qui s'est déroulé en janvier dernier, a bénéficié d'une audience encore en augmentation (Fig. 1). Cette session était dévolue aux présentations théoriques, qui sont retranscrites dans ce numéro. Jean-Michel Godchaux a dressé l'état des lieux de cette technique en France en 2014, établi sur les bases d'un questionnaire (208 réponses), des chiffres de la CNAM et des constructeurs d'échoendoscope [1]. Cette étude renouvelée tous les quatre ans donne un éclairage intéressant sur l'évolution de cette technique, que l'on promettait à une mort rapide lors de l'avènement de l'IRM. L'activité est en constante augmentation avec près de 85224 procédures en $2014(+25 \%$ en quatre ans) (Fig. 2). On dénombre 330 centres équipés d'au moins un échoendoscope, soit $30 \%$ des services d'endoscopie. Les indications biliopancréatiques restent majoritaires, soit $80 \%$ des actes. De même, les ponctions guidées ont plus que doublé entre 2007 et 2014, et représentent actuellement $20 \%$ des procédures. De manière surprenante, plus de la moitié des biopsies sont encore précédées par un examen radial. Or, il est désormais admis que l'échoendoscopie linéaire a une performance diagnostique équivalente pour la recherche de calculs biliaires, pour le staging tumoral œsophagien ou rectal, voire supérieure pour l'exploration du pancréas (pancréatite chronique, tumeur, screening), rendant cet examen préalable probablement inutile [2-6]. L'utilisation exclusive d'un appareil linéaire lors d'une indication de ponction devrait donc se généraliser dans le futur. Par ailleurs, la formation des échoendoscopistes français est effectuée dans le cadre du diplôme interuniversitaire dans près de $50 \%$ des cas, et il faut remercier pour ce travail Marc Barthet qui coordonne ce diplôme avec énergie depuis plusieurs années désor-

\section{E. Bories $(\bowtie)$}

Unité d'exploration médicochirurgicale oncologique,

Institut Paoli Calmettes, 232 boulevard de Sainte-Marguerite,

F-13273 Marseille cedex 09

e-mail : erwan.bories@outlook.fr mais. La formation continue est réalisée par une participation régulière au congrès annuel du Club francophone pour presque $80 \%$ des échoendoscopistes. Toutefois, l'enseignement pratique en échoendoscopie thérapeutique reste problématique actuellement et des circuits dédiés devront être mis en place, en s'inspirant des structures développées pour la cholangiopancréatographie rétrograde.

Le paysage de la coloscopie en France est évoqué au travers des résultats de l'enquête SFED 2015 [7]. Il faut noter que le nombre de participants à cette étude est en diminution par rapport à l'an dernier. Ceci est dommageable, car l'obtention de chiffres d'activités fiables et suivis dans le temps permet de fournir des données opposables à nos tutelles lors des différentes négociations.

Trois messages importants peuvent être dégagés de cette étude 2015. Le taux de complication global reste faible, mais on observe un taux d'hémorragie supérieur aux années précédentes $(0,04 \%)$, peut-être lié à une prise en charge des antiagrégants/anticoagulants inadaptée. Les préparations imparfaites diminuent grâce à la progressive généralisation des préparations splittées et le taux de coloscopies incomplètes est désormais inférieur à $2 \%$. Le taux de détection d'adénome en France est estimé à 19,2\%, encore en deçà des taux rapportés dans les dernières études publiées [8,9] avec des TDA de plus de $30 \%$ pour les hommes, $20 \%$ pour les femmes. Ces données sont à intégrer dans notre activité. En effet, les critères de qualité en endoscopie vont nous occuper et nous préoccuper de plus en plus dans les années qui viennent. Plutôt que de les subir, il convient de se les approprier et les installer durablement comme indicateurs d'efficience et de sécurité dans nos salles d'examens et mettre en place les procédures d'amélioration adaptées.

Bonne lecture de ce nouveau numéro d'Acta Endoscopica.

Liens d'intérêts : L'auteur déclare ne pas avoir de lien d'intérêt. 


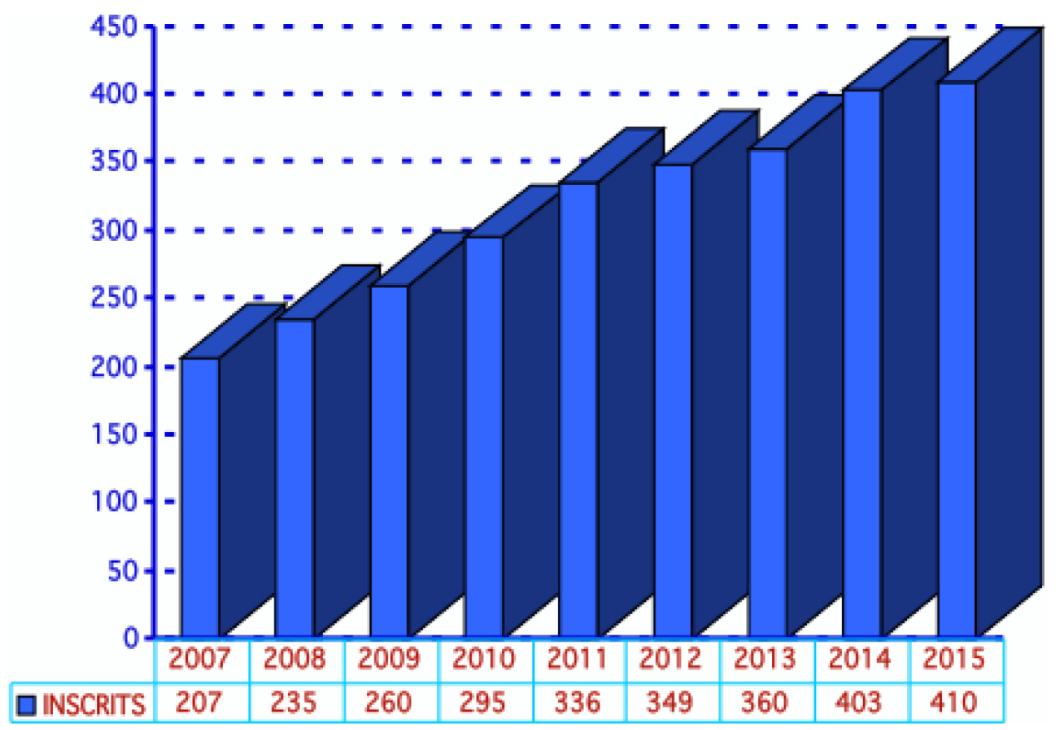

Fig. 1 Inscrits au congrès du Club francophone d'échoendoscopie [1]

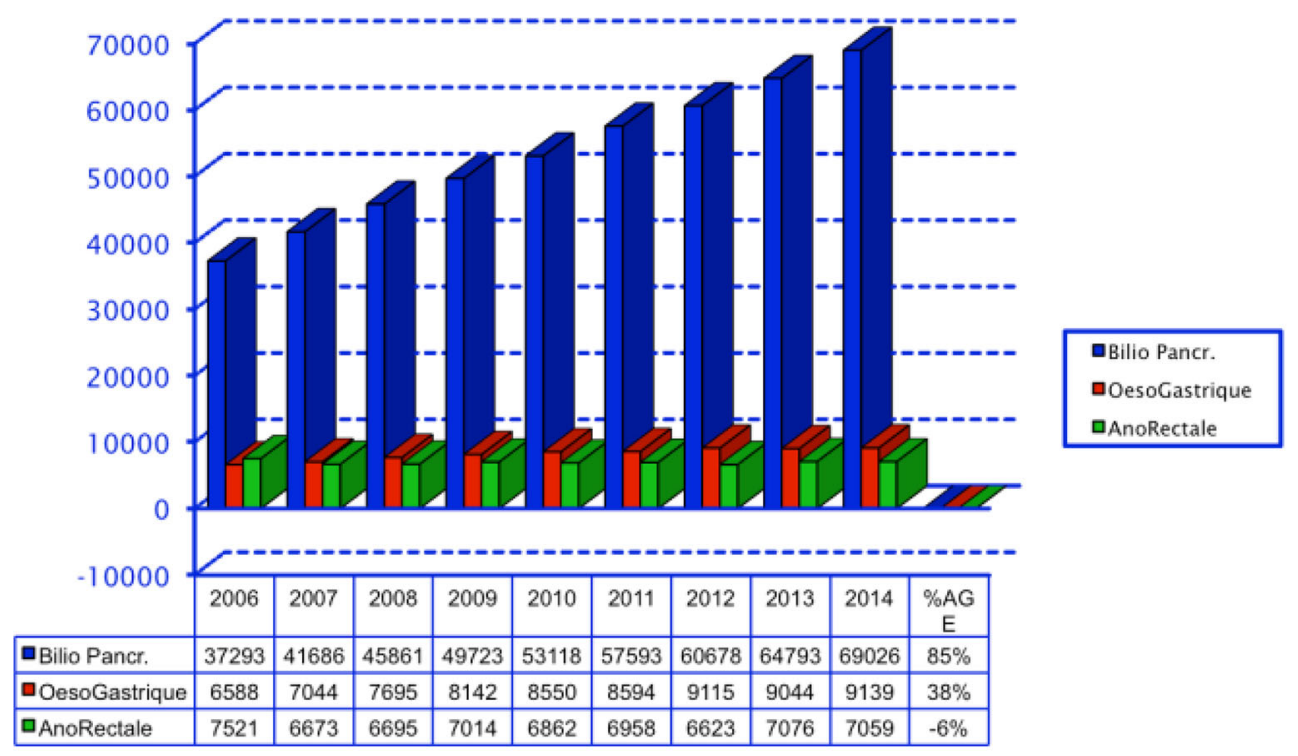

Fig. 2 Indications des échoendoscopies en France [1]

\section{Références}

1. Godchaux JM. État des lieux de l'échoendoscopie en France. CFE 2016. Données non publiées.

2. Marone P, de Bellis M, Avallone A, et al. Accuracy of endoscopic ultrasound in staging and restaging patients with locally advanced rectal cancer undergoing neoadjuvant chemoradiation. Clin Res Hepatol Gastroenterol 2011;35:666-70.

3. Shin EJ, Topazian M, Goggins MG, et al. Linear-array EUS improves detection of pancreatic lesions in high-risk individuals: a randomized tandem study. Gastrointestinal Endoscopy 2015;82:812-8.

4. Kaneko M, Katanuma A, Maguchi H, et al. Prospective, randomized, comparative study of delineation capability of radial scanning and curved linear array endoscopic ultrasound for the pancreaticobiliary region. Endosc Int Open 2014;2:E160-70.
5. Stevens T, Zuccaro G, Dumot JA, et al Prospective comparison of radial and linear endoscopic ultrasound for diagnosis of chronic pancreatitis. Endoscopy 2009;41:836-41.

6. Xu W, Liu Y, Pan P, et al. Prior Radial-Scanning Endoscopic Ultrasonography Training Did Not Contribute to Subsequent LinearArray Endoscopic Ultrasonography Study Performance in the Stomach of a Porcine Model. Gut Liver 2015;9:353-7.

7. Bernardini D, Robaszkiewicz M, Canard JM, et al. La coloscopie en 2015 : de la préparation au diagnostic. Résultats de l'enquête annuelle de la Société Française d'Endoscopie Digestive. Acta Endoscopica 46:257-65

8. Rex DK, Schoenfeld PS, Cohen J, et al. Quality indicators for colonoscopy. Am J Gastroenterol 2014;110:72-90.

9. Brenner H, Altenhofen L, Kretschmann J, et al. Trends in Adenoma Detection Rates During the First 10 Years of the German Screening Colonoscopy Program. Gastroenterology 2015;149:356-66.e1. 